\title{
WALL SLIP PHENOMENON IN HIGHLY CONCENTRATED IONIC LIQUID-BASED MAGNETORHEOLOGICAL FLUIDS
}

\author{
A. Gómez-Ramírez ${ }^{(* *)}$, M. T. López-López ${ }^{+}$, F. González-Caballero ${ }^{+}$and J. D. G. \\ Durán $^{+}$
}

(**)Instituto de Ciencia de Materiales de Sevilla (CSIC-Universidad de Sevilla), Avda. Américo Vespucio, 49, Sevilla, 41092, Spain.

${ }^{+}$Department of Applied Physics, Faculty of Sciences, University of Granada, Avenida Fuentenueva s/n, 18071 Granada, Spain.

\section{E-mail: anagr@ugr.es}

\begin{abstract}
Ionic liquids (IL) have been recently proposed as carrier for magnetorheological (MR) fluids. Their special properties, such as low vapor pressure and high thermal stability, make IL a perfect dispersing media to increase the broad range of technological applications that magnetorheological fluids already have. It has been just reported that using IL as carrier in MF fluids an improvement in the colloidal stability and suspension redispersibity is obtained. In this work the magnetorheological behavior of highly concentrated suspensions in IL is studied. Two kinds of suspensions were analyzed: using an ionic liquid of low conductivity and a classical mineral oil as carriers. In both cases silica-coated iron microparticles were used as solid phase, being the solid volume concentration of $50 \%$ vol. A complete magnetorheological analysis focused on the wall slip phenomenon was performed. Viscositmetry and oscillatory experiment were carried out. In order to study wall slip effects, all experiments were performed with a plate-plate system, using both smooth and rough measuring surfaces. An interesting effect of wall slip phenomenon was observed when experiments were performed using smooth surfaces. The novelty of this paper is based on two factors, the use of IL as carrier to prepare MR fluids and the analysis of wall slip phenomena.
\end{abstract}

\section{INTRODUCCTION}

Magnetorheological fluids are suspensions of magnetic microparticles in a carrier liquid that present the possibility of modifying their rheological properties by applying a magnetic field [1-4]. Their most important characteristic, called magnetorheological (MR) effect, is the appearance of a yield stress under magnetic field application [5]. Because of the broad range of technological applications that these materials have, which go from electronic devices [6] or shock absorber systems [7-9] to their use in biomedicine [10-12], intense research efforts have been paid to MR fluids. In the last decade an increasing number of works focused on solving the problems that MR fluids recently present (particle aggregation, long-time stability, redispersibility of magnetic particles, higher MR effect, etc.) have been published [13-19]. Nevertheless, some these problems still remain unsolved, and the development of new highly stable MR fluids with a remarkable MR effect is nowadays a priority research field.

Using Ionic Liquids (IL) as carrier liquid for MR fluids has been recently proposed to obtain more stable MR fluids [20]. IL liquids are substances entirely composed by ions which are in the liquid state at ambient temperature [21-22]. These fluids present a 
large number of advantages for their use as carriers in MR fluids: low vapor pressure, non-flammability, high thermal stability, no-corrosive nature and biodegradability [2326]. Although some characteristic of IL are still unknown [27], in ref. [20] it was clearly pointed out an improvement in the stability of the MR suspensions when IL were used as liquid carrier, without the necessity of using surfactant or others additives to stabilize the suspensions $[28,29]$. In a previous work [30] we demonstrated that a good colloidal stability and redispersibility was reached when a low conductivity ionic liquid was used as carrier liquid and silica coated iron particles as solid phase. Furthermore, it was observed that when these materials were used to prepared MR suspensions more ordered magnetic structures were formed under magnetic field application. Based on the above it could be stated that IL represent an promising alternative to improve the properties of MR fluids, though not too much work have been published on this topic up to now [31-36].

In this work, the MR behavior of these new IL-based MR fluids is studied. Steady state and oscillatory measurements are performed. In both cases, the MR experiments are focused on the study of the wall slip phenomenon. Wall slip occurs because of the displacement of the disperse phase away from the solid boundaries, leaving a lowerviscosity layer of liquid (named slip layer) close to measuring surfaces [37, 38]. Wall slip may alter the determination of the rheological parameters, so, the knowledge of the slip layer properties and wall slip effects is quite important in order to characterize correctly the rheological behaviour of the suspensions. It is well known that wall slip provokes an underestimation of suspension viscosity and even it could give false thixotropy [38]. The use of measuring systems with rough surfaces has been, up to now, the best option to avoid wall slip effects [39-41]. So, in this work, a plate-plate measure system with two types of surfaces (smooth and rough) is used. All the results are compared with those obtained for a classical MR suspension, in which the liquid carrier is a typical mineral oil.

Although wall slip phenomenology and its effects are well known since years [37, 39, 42-46], studies regarding their effects in MR suspensions have not been published. This work represents, up to our knowledge, the first preliminary analysis about wall slip in highly stable MR fluids. It is worth mentioning that scientific community should become aware about the importance of such phenomenon; in order avoid its undesirable effects. Future works about this topic are expected in the next years.

\section{EXPERIMENTAL METHODS}

\subsection{SUSPENSION PREPARATION}

The MR analysis was performed for suspensions containing $50 \%$ vol. of silica coated iron particles (Fe-CC particles) supplied by Basf, Germany. The solid concentration is so high because the higher it is, the more important wall slip effects are. Wall slip effects will be more important as the solid volume concentration in the suspensions and particle size increases, although the slip layer in these cases was thinner [37].

The mean diameter of the particles used is $1.4 \pm 0.6 \mu \mathrm{m}$ and their density is 4.1 $\mathrm{g} / \mathrm{m}^{3}$. Their saturation magnetization is $870.9 \pm 1.4 \mathrm{kA} / \mathrm{m}$. These particles were used to prepare two kinds of suspensions, using an ionic liquid or a mineral oil as dispersing media. As ionic liquid, 1-ethyl-3-methylimidazolium diethylphosphate, called IL-EMD from now, was used (Viscosity $317 \pm 16 \mathrm{mPa} \cdot \mathrm{s}$ and density $1.14 \mathrm{~g} / \mathrm{cm}^{3}$ ). It was chosen because it has been recently proved that the suspensions in this carrier present a higher 
stability and a lower aggregation degree that when a mineral oil or other ionic liquids are used [30]. The conductivity of the IL-EMD is $0.66 \mathrm{mS} / \mathrm{cm}$. To compare the results with those obtained for a conventional MR fluid, a suspension in a typical (MO) mineral oil (density and viscosity at $20^{\circ} \mathrm{C}: 0.85 \mathrm{~g} / \mathrm{cm}^{3}$ and $39.58 \pm 0.16 \mathrm{mPa} \cdot \mathrm{s}$, respectively) was also studied. Suspensions were prepared in the same way that it is described in ref. [30]. It is interesting to note that IL-based suspensions must be manipulated with special care, since IL tend to absorb water from atmosphere [33, 24].

\subsection{RHEOLOGICAL EXPERIMENTS}

To carry out the MR analysis a controlled stress magnetorheometer (MCR300 Physica-Anton Paar) was used. The measuring system employed was a plate-plate geometry with a gap of $0.35 \mathrm{~mm}$. All the measurements were performed at $25^{\circ} \mathrm{C}$. Although the width gap has not been varied in this work it should be mentioned that as narrower it is, the effect of the slip layer will be higher [38]. Both, steady state and oscillatory experiments were carried out. Before each measurement, the suspension was subjected to a shear rate ramp from 0 to $100 \mathrm{~s}^{-1}$ during a minute. After that the suspension was kept at rest during $30 \mathrm{~s}$. At the beginning of this waiting time the magnetic field was applied in those cases in which it was necessary. In the case of steady state measurements, a logarithmic shear rate ramp, from 0 to $500 \mathrm{~s}^{-1}$, was applied and the corresponding viscosity and shear stress were measured. For the oscillatory study, we applied an oscillatory stress with amplitude in the range 0.1-500 $\mathrm{Pa}$, and a frequency of $1 \mathrm{~Hz}$. The evolution of the viscoelastic moduli was obtained as a function of the stress amplitude. Furthermore, the evolution of the viscoelastic moduli with the frequency was also analyzed. The frequency was varying in the range $25-100 \mathrm{~Hz}$ with a constant stress applied of $2 \mathrm{~Pa}$, which belongs to the viscoelastic linear region (VLR) for all the suspensions studied. For both, steady state and oscillatory measurements, the magnetic field applied was varied in the range $0-39.9 \mathrm{kA} / \mathrm{m}$.

To apply the magnetic field a special coil was designed. The magnetic field generated by the magnetorheometer device is not completely homogenous in the measuring region [47, 48], since the sample is located just on the coil. As suspensions have a really high magnetic particle concentration, the magnetic field gradient throws the suspension out the plates. In our case, the special coil designed was situated just around the sample, in this way the measuring region occupied just the centre of the coil. Using this configuration the magnetic field was more homogeneous in the measuring region and no expulsion was observed.

As it is explained above, this work is focussed in the study of wall slip, so, to avoid the influence of such phenomena in the determination of the MR parameters, measuring plates with two kinds of surfaces, smooth and rough, were used to carry out the MR experiments. In figure 1 a sketch of the rough geometry can be observed. The pitted size is $0.5 \mathrm{~mm}$.

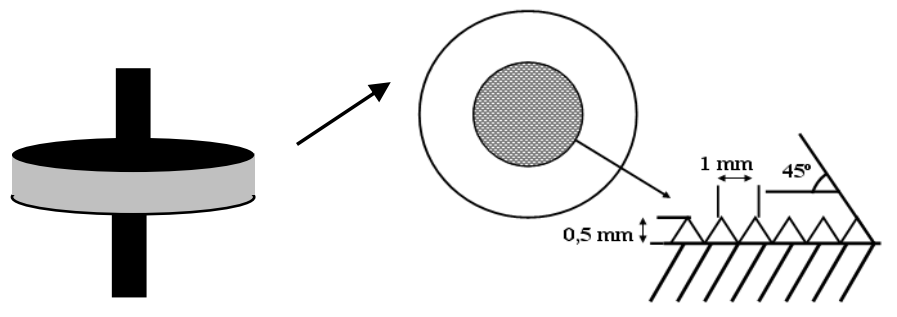

Figure 1. Sketch of the rough geometry. 


\section{RESULTS AND DISCUSSION: MAGNETORHEOLOGICAL ANALYSIS}

\subsection{VISCOSIMETRY}

\subsubsection{Viscosity analysis}

Firstly, we are going to analyze the effect of wall slip phenomena in the viscosity of the suspensions prepared. In figure 2 the viscosity of both kinds of suspensions is shown as a function of the shear rate in the absence (figure $2 a$ ) and in the presence (figure $2 b$ ) of a magnetic field applied. Full and empty symbol correspond with smooth and rough surfaces respectively. As we can observe in that figure, for both suspensions studied (Fe-CC in MO and Fe-CC in IL-EMD), the obtained viscosity was higher when it was measured by means of plates with rough surfaces than when plates with smooth surfaces were used. Curves obtained with smooth surfaces presented changes in their curvature, while these changes are not presented using rough surfaces. This effect happens at low shear rate values, before the flow of the suspensions and before the decrease of the viscosity due to microstructural changes in the suspensions [37].
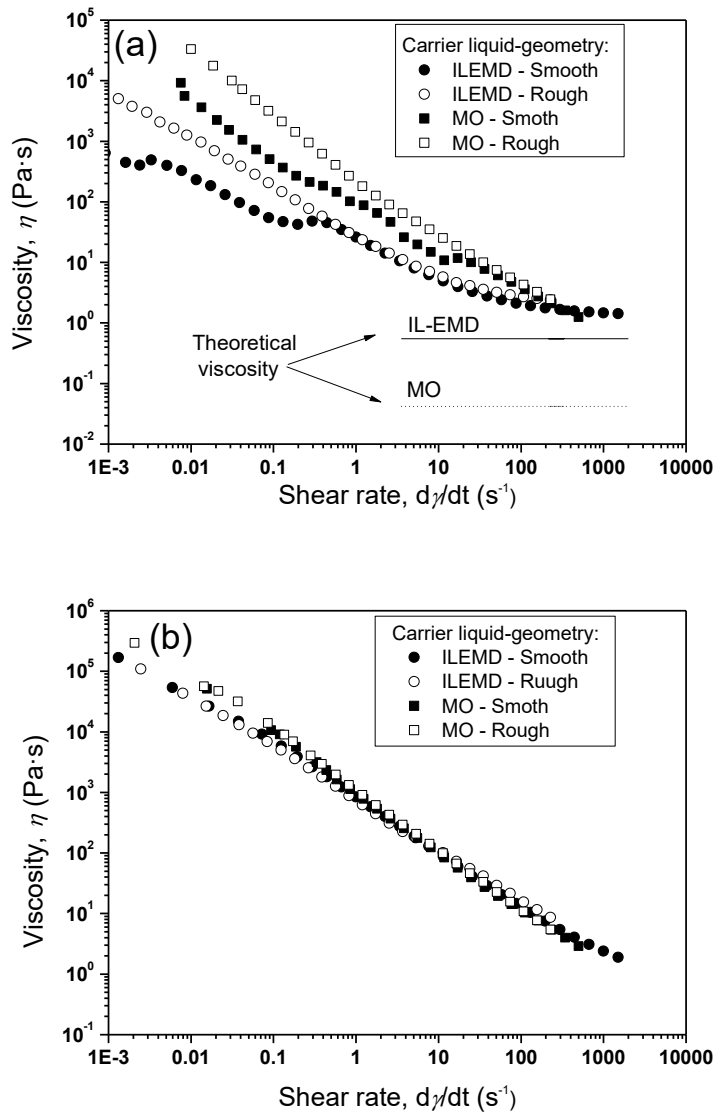

Figure 2. Viscosity as a function of the shear rate for suspensions of Fe-CC $(\phi=50 \%$ vol.). The carrier liquids and the geometries used are indicated in the graph. (a) $\mathrm{H}_{\text {applied }}=$ $0 \mathrm{kA} / \mathrm{m}$, (b) $\mathrm{H}_{\text {applied }}=39.9 \mathrm{kA} / \mathrm{m}$. The lines in (a) represent the K-D prediction (see equation 1).

The viscosity underestimation (using smooth surfaces) is a consequence of the existence of wall slip. This phenomenology is a result of the formation of a slip layer 
near the measuring surfaces $[39,40]$. In this layer the particle concentration is lower and then the viscosity measured is also lower than its actual value with respect to the suspension bulk. Figure 3 shows a sketch of how formation of slip layer takes place. Some interactions (hydrodynamic, viscoelastic, steric) are restricted due to the presence of the wall, and then the slip layer is formed.

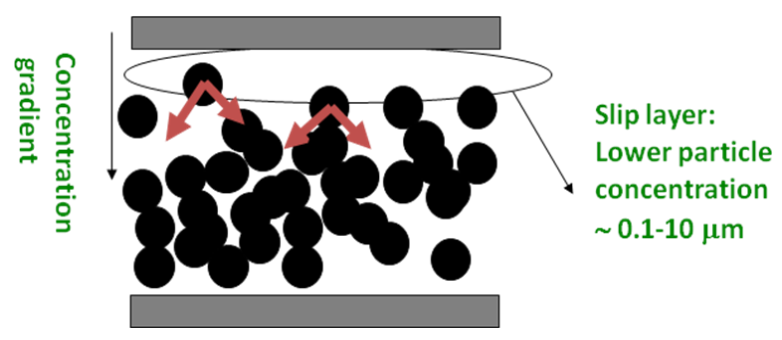

Figure 3. Sketch of slip layer formation.

Furthermore, it is important to take into account that slip layer formation is enhanced by the effect of gravity, since plate-plate geometry is being used, and particles are supposed to leave the region close to the upper plate. In ref. [39] it was demonstrated that even at the lower time involved in a typical rheological experiment, the effect of the gravity on the slip layer can be noticeable.

On the other hand, in figure $2 b$ it can be observed that under magnetic field application the differences between the viscosity curves obtained for different suspensions and measuring surfaces disappear, and all the curves overlap. The slip layer disappears due to the formation of magnetic field-induced particles structures in the suspensions. The interaction between such particles structures and the plates of the measuring system is considerably high [38]. This is the reason because, when a magnetic field is applied, approximately the same results are obtained using the smooth or rough surfaces.

Moreover, in figure 2a two lines corresponding to the theoretical prediction for the viscosity of the suspensions following the Krieger-Dougherty (K-D) equation have been added [49]:

$$
\eta=\eta_{s}\left(1-\frac{\phi}{\phi_{m}}\right)^{-[\eta] \phi_{m}}
$$

where $\phi_{m}$ is the maximum packing solid volume fraction (for hard spheres: 0.63-0.64) and $[\eta]$ is the intrinsic viscosity (2.5 for spheres) [50]. As we can observe (figure 2a), the experimental viscosity obtained for the suspension in IL-EMD is closer to the theoretical prediction than that of the suspension in MO. This fact is in agreement with the previous results [30] where it was demonstrated the higher aggregation degree of the latter suspension.

\subsubsection{Magnetorheological analysis}

Flow curves were measured for both kind of suspensions and surfaces, in the absence and in the presence of magnetic field with different intensities. Although the representations of the shear stress vs. shear rate are not shown here for brevity, it is important to note that both suspensions (Fe-CC in $\mathrm{MO}$ and $\mathrm{Fe}-\mathrm{CC}$ in IL-EMD) presented a typical MR behavior: the yield stress increased with the intensity of the applied magnetic field. Furthermore, it is important to take into account that at zero field and low shear rate, changes in the curvature of the flow curve are observed when plates 
with smooth surfaces were used to perform the measurement (similar that in the viscosity determination). This effect is also a consequence of the presence of the wall slip phenomena. To analyze this effect more deeply in figure $4 \mathrm{a}$ and $4 \mathrm{~b}$ the static and the dynamic yield stress are plotted as a function of the magnetic field applied. Equals as in figure 2 the results are shown for both surfaces and geometries studied. The values of the static yield stress were obtained from double-logarithmic plots of the flow curves; while the values of the dynamic yield stress were obtained by fitting to the flow curves the Bingham model [50].
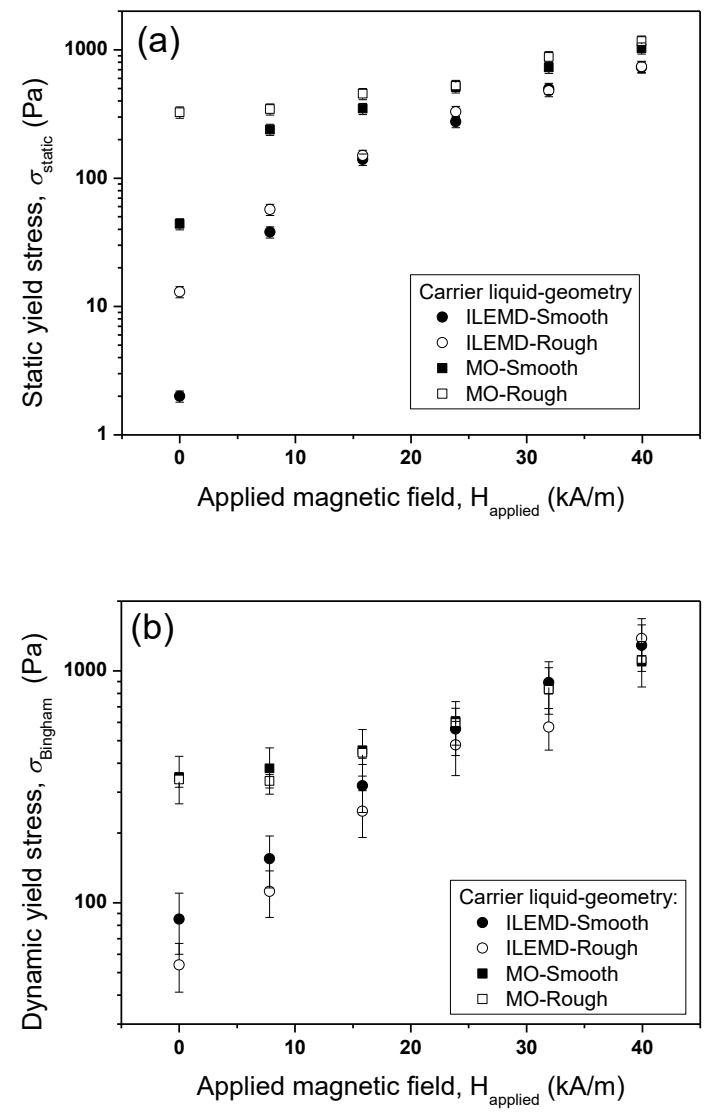

Figure 4. Static yield stress (a) and dynamic yields stress (b) as a function of the magnetic field intensity for suspensions containing Fe-CC particles ( $\phi=50 \%$ vol.). The carrier liquids and the geometries used are indicated in the graph.

The most interesting aspects in figure $4 \mathrm{a}$ are the differences obtained when rough plates were used instead of smooth plates. As it can be observed, such differences are more important in the absence of the field or at low magnetic fields $(<15 \mathrm{kA} / \mathrm{m})$. For example, in the absence of magnetic field, when rough surfaces were used, the obtained static yield stress increases (with respect to that obtained when smooth surfaces were used) from 44 to $326 \mathrm{~Pa}$ and from 2 to $12 \mathrm{~Pa}$ for the suspensions in MO and IL-EMD, respectively. In these cases, the slip layer had a notable effect. The lower particle concentration in the slip layer provoked an underestimation of the static yield stress, when measurements were performed using smooth surfaces. Furthermore, when the magnetic field intensity increased such differences disappeared progressively, due to the formation of magnetic structures that eliminated the slip layer. Finally, in figure 4a it can be observed that, even in the absence of applied magnetic field, the suspension in MO developed a high static yield stress: Values of 326 and $44 \mathrm{~Pa}$ were obtained with 
rough and smooth surfaces, while in the case of IL-EMD suspension these values are of 13 and $2 \mathrm{~Pa}$. This result is in agreement with our previous observations [30] and it is a consequence of the higher aggregation degree of suspension in MO.

On the contrary, in figure $4 \mathrm{~b}$ the most relevant feature is the fact that there are not differences between the curves obtained using different measuring surfaces (smooth and rough). This is quite logical, since the dynamic yield stress represents the point at which all the structures induced by the magnetic field are broken [51]. When the suspension flows, it is expected that the slip layer does not exist, and thus there will not be differences between both kinds of geometries [37]. Here, it is also important to point out the great interest of highly concentrate suspensions in applications where it is necessary an important MR response. When a small magnetic field is applied (around $40 \mathrm{kA} / \mathrm{m}$ ), a dynamic yield stress of $1000 \mathrm{~Pa}$ can be obtained with suspensions containing a $50 \%$ vol. of iron particles.

\subsection{OSCILOMETRY}

The MR behavior of the suspensions in oscillatory regimen was also studied. To be precise, the evolution of the viscoelastic moduli with the amplitude of the oscillatory stress and with the frequency was analyzed. The study was performed in the absence and in the presence of applied magnetic field, and for both kinds of suspensions (Fe-CC in $\mathrm{MO}$ and Fe-CC in IL-EMD) and measuring surfaces (smooth and rough). The dependence of the storage $\left(G^{\prime}\right)$ and loss $\left(G^{\prime \prime}\right)$ moduli with the amplitude of the stress for the different magnetic field applied is not shown because of brevity, but it must be remarked that the behavior obtained in all the cases was the typical of a viscoelastic fluid. The viscoelastic moduli present an initial plateau, which corresponds to the viscoelastic linear region (VLR) followed by a sharp decrease, that represents the beginning of the non-linear viscoelastic region. The characteristic stress at which this change happens, because of the rupture of the three-dimensional structures present in the suspension, is named critical stress, and here will be denoted by $\sigma_{\mathrm{c}}$. As the magnetic field was increased, the magnetic structures induced in the suspensions were stronger. As a consequence, the critical stress and the values of $G^{\prime}$ and $G$ '' increased too. It is also interesting to note that the storage modulus was always higher than loss modulus, which means that the suspensions had a dominant elastic response. Nevertheless, it is important to take in account that the viscous response of the suspensions was not negligible. There are different theories for explaining the increase of the loss modulus with the magnetic field in MR fluids. In ref. [52] the authors proposed that the chains of particles that are not in contact with the two plates of the measuring system are responsible for the high viscous response of the suspensions. On the other hand, authors in ref. [53] proposed that such behavior is a consequence of the non-affine movement of the particles inside the magnetic structures.

Figure 5 shows the values of $G^{\prime}$ obtained for each suspension (Fe-CC in $\mathrm{MO}$ and Fe-CC in IL-EMD) and measuring surfaces (smooth and rough) as a function of the oscillatory stress amplitude, in the absence (figure 5a) and the presence (figure 5b) of applied magnetic field $(39.9 \mathrm{kA} / \mathrm{m})$. As we can observe, in the absence of field, the values of $G^{\prime}$ obtained using the rough surfaces are higher that those obtained using smooth surfaces. In the VLR, for the suspension in MO the mean value of $G$ ' is around $730 \mathrm{kPa}$ when rough surfaces are used, while this value is of approximately $30 \mathrm{kPa}$ when smooth surfaces are used. When a magnetic field is applied (figure 5b) the differences between both kinds of surfaces tend to minimize. The reasons that explain 
this behavior are the same that we exposed in the case of steady state measurements: in the absence of magnetic field the existence of a slip layer provoked that the viscoelastic moduli were underestimated; in the presence of field such difference disappeared progressively as a consequence of the elimination of the slip layer. Similar results were obtained for the loss modulus, $G$ ', but there are not shown here for brevity.
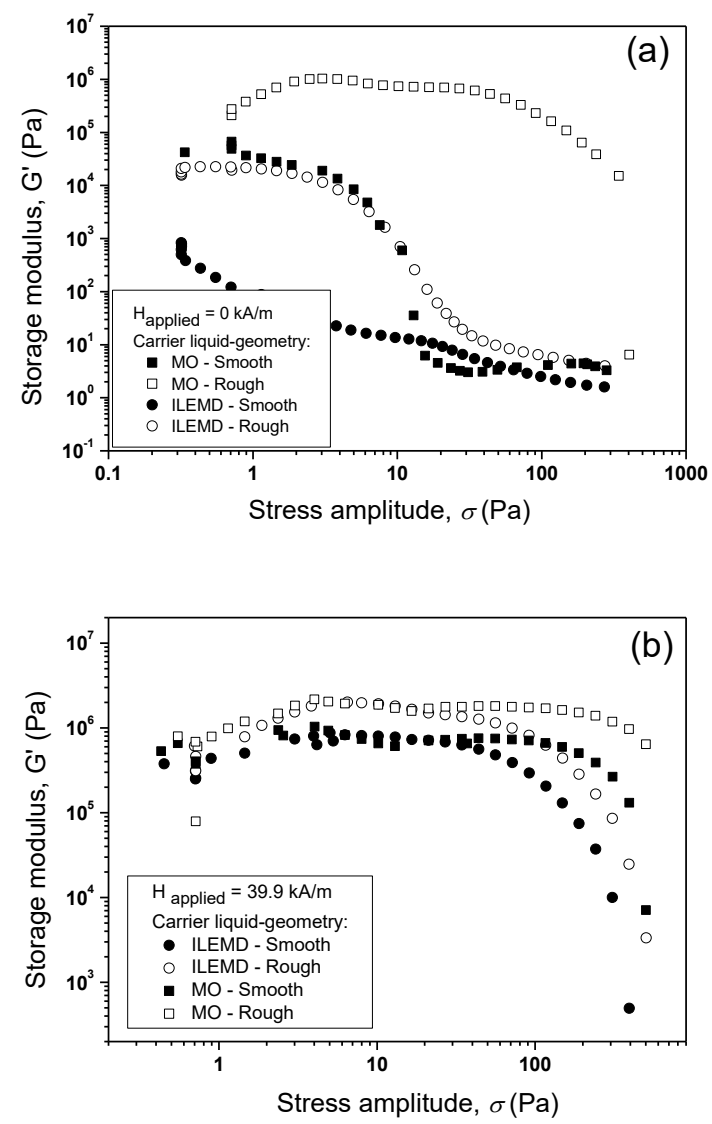

Figure 5. Storage modulus, G', as a function of the oscillatory stress amplitude (frequency $1 \mathrm{~Hz}$ ) for suspensions of Fe-CC particles $(\phi=50 \%$ vol.). The carrier liquids and measuring surfaces used are indicated in the graph. Intensity of the magnetic field applied: (a) $\mathrm{H}_{\text {applied }}=0 \mathrm{kA} / \mathrm{m}$; (b) $\mathrm{H}_{\text {applied }}=39.9 \mathrm{kA} / \mathrm{m}$.

In order to get a better picture of the effects of wall slip, we can analyze the averaged values of the viscoelastic moduli in the VLR as a function of the intensity of the applied magnetic field, for both kinds of measuring surfaces and suspensions studied. Figure 6 shows such plot for the storage (figure 6a) and loss (figure 6b) moduli. In this figure we can observe that, for the suspension in IL-EMD, both moduli (with rough and smooth geometries) increase as the magnetic field is raised, due to the hardening of the magnetic structures. On the contrary, in the case of suspensions in MO the increase is not so clear, due to the high aggregation degree of this suspension. In figure 6 we can also observe the effect of wall slip. At low field, the averaged values of $G$ ' are higher when the rough geometry is used. As it was the case for the static yield stress, as the magnetic field increases such differences disappear. By comparing the results obtained in figure $6 \mathrm{a}$ and $6 \mathrm{~b}$ it can be concluded that suspensions present a dominant elastic response, since the values of the storage modulus are higher than values of the loss modulus. 

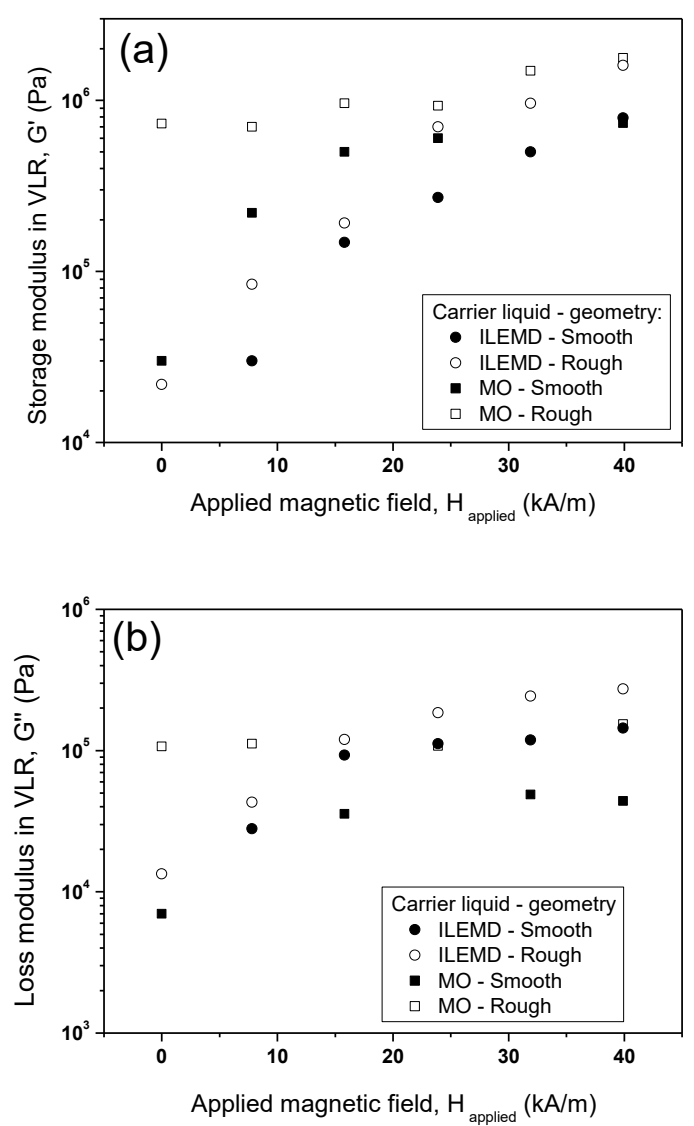

Figure 6. Storage (a) and loss (b) moduli (average in the VLR) as a function of the magnetic field for suspensions of Fe-CC particles ( $\phi=50 \%$ vol.). The carrier liquids and geometries employed to carry out the measurements are indicated in the graph.

To have more information about the oscillatory regime, the values of the critical stress as a function of the applied magnetic field for both kind of suspensions and measuring surfaces are shown in figure 7 . In this case, equal that happened in the case of the static yield stress, the values obtained with the rough surfaces are higher than those obtained with of smooth surfaces. The same explanation given before stands here. Using smooth surfaces the critical stress is underestimated. For the suspension in MO in the absence of field, the critical stress is more than one order of magnitude higher when rough surfaces are used instead of smooth ones. Apart from this, only two other features are relevant in figure 7 . The first one is that the curves tend to minimize their differences as the magnetic field increases (the slip layer progressively disappears); the second one is that the critical stress values obtained in the case of the suspensions in MO are higher than those corresponding to the suspensions in IL-EMD, something that is also due to the high degree of aggregation of the former suspension. 


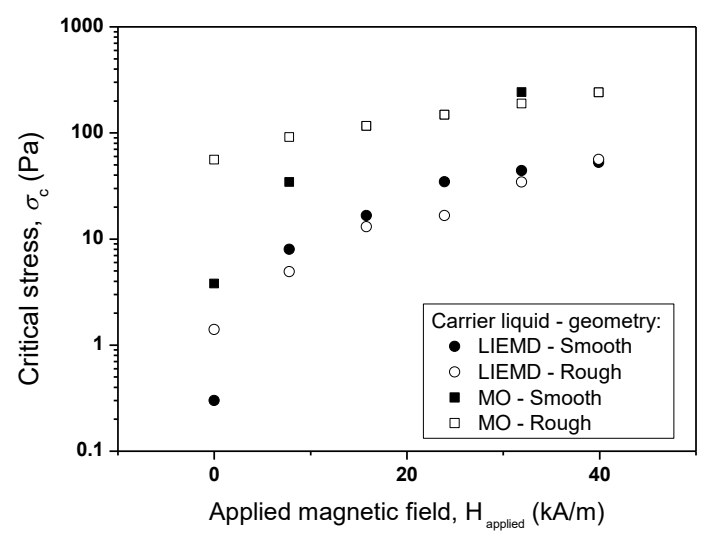

Figure 7. Critical stress, $\sigma_{c}$, as a function of the applied magnetic field for suspensions of Fe-CC particles ( $\phi=50 \%$ vol.). The different curves correspond to the carrier liquids and measuring surfaces indicated in the graph.

Finally, to complete oscillatory analysis, the evolution of the storage and loss moduli with the frequency of the applied stress was analyzed. The dependence of the viscoelastic moduli with the frequency follows, in most of the cases, a typical Maxwell behaviour; at low and medium frequency the storage modulus increases regularly, while it reaches a plateau at high frequency. On the contrary, the loss modulus typically presents a maximum at medium frequencies. In the case of electrorheological fluids, it has been demonstrated that the storage modulus is independent of the frequency at high electric field [54-56] and, at least, one order of magnitude higher than the loss modulus. Figure 8 shows the evolution of the storage modulus as a function of the frequency for the suspension of Fe-CC particles in the IL-EMD obtained by applying different magnetic field. Curves shown in figure 8a have been obtained with smooth measuring surfaces while figure $8 \mathrm{~b}$ shows those obtained using the rough ones. As can be observed, there is an increase of the storage modulus with the frequency, independently of the measuring surface used. Furthermore, the final plateau is not reached at high frequencies. For the highest field the increase of $G^{\prime}$ is less important than the obtained at low field or in the absence of magnetic field. Similar tendencies were obtained for the loss modulus, not shown here for brevity. Nevertheless, two important facts should be mentioned. The first one is that the increase of the $G^{\prime}$ ' with frequency is not as important as in the case of G', and the second one is that in any case the G' expected maximum at medium frequencies was observed. It is worth mentioning that the values of G', are lower that G' values, pointing out, again, the higher elastic response of our suspension. Similar tendencies were obtained for Fe-CC in MO suspension. 

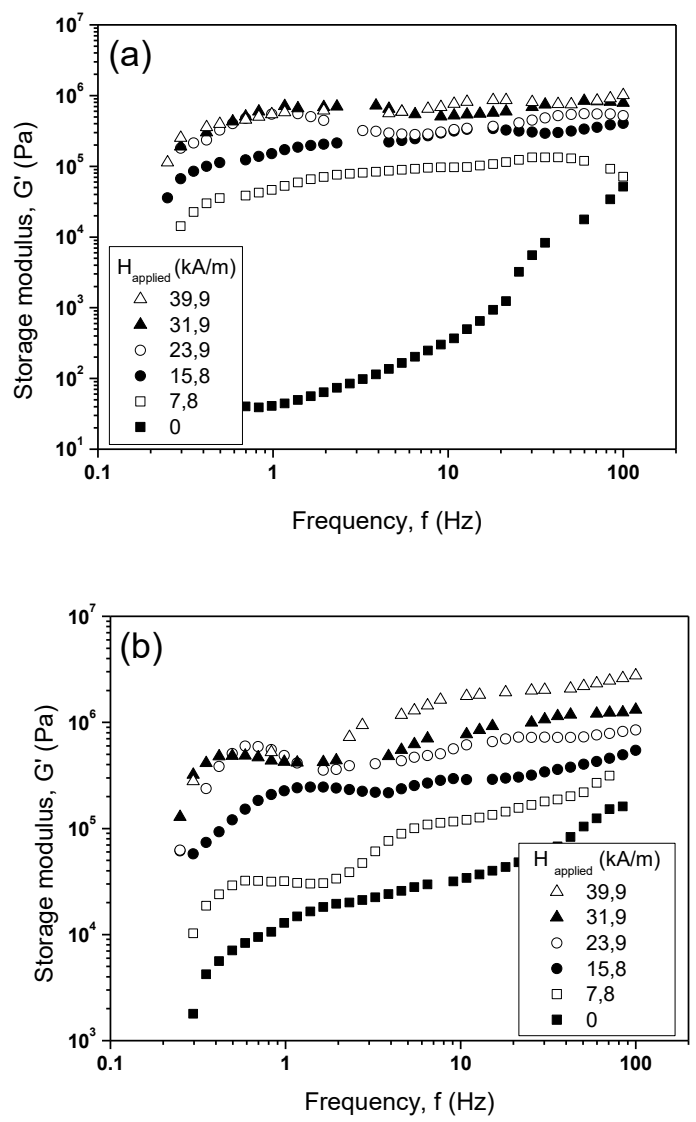

Figura 8. Storage modulus as a function of the frequency (oscillatory stress amplitude 2 $\mathrm{Pa}$ ) for suspensions of Fe-CC particles in IL-EMD. (a) Smooth surfaces (b) rough surfaces. Magnetic field intensity is indicated in the graph.

To compare the response of suspensions in MO or ILEMD, in figure 9 the evolution of the storage modulus is plotted as a function of the frequency for both kinds of measuring surfaces in the absence (figure 9a) and in the presence (figure9b) of external magnetic field. As we observe, in the frequency domain, when smooth surfaces are used the storage modulus is also underestimated, both under magnetic field applied and in the absence of field. Similar results were obtained in the case of loss modulus. Finally, differences obtained with different measuring surfaces are higher for the suspension in IL-EMD that for suspension in MO, which confirms one more time the higher aggregation state of suspension in MO. This fact is especially important in the absence of magnetic field and low frequencies (figure 9a). In figure 9 can be observed that under magnetic field application the differences between curves disappear, as a consequence of the elimination of the slip layer. In the case of the loss modulus the results obtained were similar that for the storage modulus. 

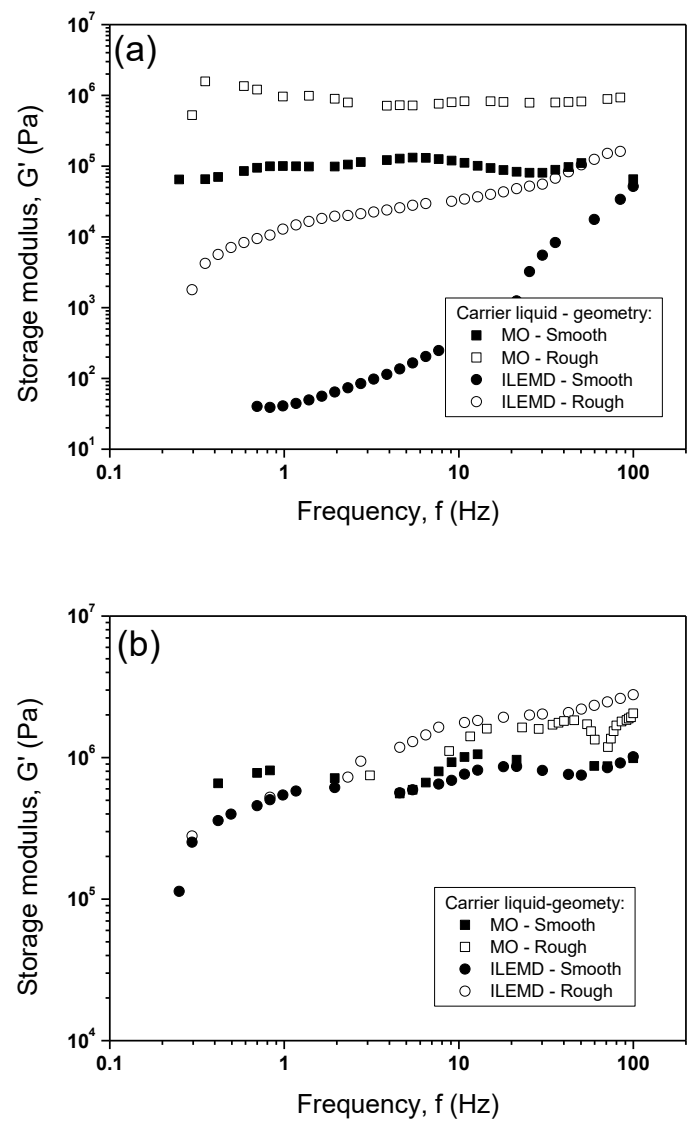

Figure 9. Storage modulus as a function of the frequency (oscillatory stress amplitude 2 $\mathrm{Pa}$ ) for suspensions of Fe-CC particles in IL-EMD and MO. Measuring surfaces used are indicated in the graphs. (a) $\mathrm{H}_{\text {applied }}=0 \mathrm{kA} / \mathrm{m}$; (b) $\mathrm{H}_{\text {applied }}=39,9 \mathrm{kA} / \mathrm{m}$.

\section{CONCLUSIONS}

In this work the magnetorheological behavior of highly concentrated IL-based MR fluids has been analyzed. The MR analysis has been focused on the study of wall slip phenomena. IL have been used as carrier because it provides more stable colloidal suspensions than using a classical mineral oil [20,30]. Highly concentrated suspensions with silica-coated iron particle in the micron-size have been used because big particles and high concentrations bring more important slip effects [37]. Both viscosimetry and oscillometry measurements have been carried out. All the experiments have been performed using two kind of measuring surfaces: smooth and rough plates. The results have been compared with those obtained for a classical suspension (with mineral oil as carrier liquid). As main conclusion of this work it can be stated that wall slip phenomenon has an important effect in the determination of the rheological parameters that characterize the magnetorheological behavior of the MR suspensions. It has been proved that using measuring geometries with smooth surfaces to perform the experiments instead of rough surfaces, an underestimation of the viscosity, the static yield stress, the viscoelastic moduli and the critical stress is obtained.

Finally, it is important to comment two important facts. Firstly, it is remarkable the great interest that IL have gained in the last years. A great scientific effort is necessary to understand the complex mechanisms that promote the stabilization of magnetic particles in this new kind of carrier liquids. On the other hand, it should be mentioned that this work represents the first preliminary study regarding wall slip effects in 
magnetorheological suspensions. Based on the conclusions herein, it is expected that scientists are aware of the importance that wall slip has in the magnetorheological characterization of the suspensions.

\section{Acknowledgments}

Financial support by Ministerio de Ciencia e Innovación (Spain) under project no. FIS2009-07321 and by Junta de Andalucia (Spain) under project nos P08-FQM-3993 and P09-FQM-4787 is gratefully acknowledged. AG-R and MTLL acknowledge financial support by Secretaría de Estado de Universidades e Investigación (MEC, Spain) through its FPU program and by Universidad de Granada (Spain), respectively.

\section{BIBLIOGRAFY}

[1] Phulé P. P. and Ginder J. M.; in Proc. 6th Int. Conference on Electro-rheological Fluids, M. Nakano and K. Koyama, ed. (World Scientific, Singapur, 1998).

[2] Ginder J. M., MRS Bull., 23, 26 (1998).

[3] Charles S. W., in Ferrofluids, S. Odenbach, ed. (Springer, Berlin, 2002).

[4] Park B. J., Fang F. F. and Choi H. J., Soft Matter, 6, 5246 (2010).

[5] Bossis G., Volkova O., Lacis S. and Meunier A., in Ferrofluids, S. Odenbach, ed. (Springer, Berlin, 2002).

[6] Ross C., Ann. Rev. Mater. Res., 31, 203 (2001).

[7] Carlson J. D., Patent N: US6151930A -2000-11-28 (2000).

[8] Wereley N. M., Hu W., Kothera C. S. and Chen P., Patent N: US2009218443 (A1). 2009-09-03 (2009).

[9] Choi K. M., Cho S. W., Jung H. J. and Lee I. W., Earthquake Engng. Struct. Dyn., 33, 723 (2004).

[10] Tartaj P., Morales M., Veintenillas-Verdaguer S., González-Carreño T. and Serna C. J., Review. J. Phys. D: Appl. Phys., 36, 182, (2003).

[11] Parthasarathy M., Ahn K. H., Belongia B. M. and Klingenberg D. J, Int. J. Mod. Phys. B, 8, 2789 (1994).

[12] Durán J. D. G., Arias J. L., Gallardo V. and Delgado A. V., J. Pharm. Sci., 97, 2948 (2008).

[13] López-López M. T., Zugaldía A., González-Caballero F. and Durán J. D. G., J. Rheol., 50, 543 (2006).

[14] López-López M. T., Vertelov G., Kuzhir P., Bossis G. and Durán J. D. G., J. Mater. Chem., 17, 3839 (2007).

[15] Odenbach S., Coll. \& Surf. A: Physicochem. Eng. Aspects, 217, 171 (2003). 
[16] De Vicente J., Vereda F., Segovia-Gutierrez J. P., Morales M. D. and HidalgoAlvarez R., J. Rheol., 54, 1337 (2010).

[17] Gómez-Ramírez A., López-López M. T., Durán J. D. G. and González-Caballero F., Soft Matter, 5, 3888 (2009).

[18] Kuzhir P., López-López M. T. and Bossis G., J. Rheol., 53, 127 (2009).

[19] Bell R. C., Miller E. D., Karli J. O., Vavreck A. N. and Zimmerman D. T., Int. J. Mod. Phys. B, 21, 5018 (2007).

[20] Guerrero-Sánchez C., Lara-Ceniceros T., Jimenez-Regalado E., Rasa M. and Schubert U. S., Adv. Mater., 19, 1740 (2007).

[21] Beyersdorff T., Schubert T. J. S., Welz-Biermann U., Pitner W., Abbott A. P., McKenzie K. J. and Ryder S., Electrodeposition from Ionic Liquids (Wiley-Vch Verlag GmbH \&Co., Weinheim, 2008).

[22] Wilkes J. S. and Zaworotko J. M., Chem. Commun., 13, 965 (1992).

[23] Armand M., Endres F., MacFarlane D. R., Ohno H. and Scrosati B., Nat. Mater., 8, 621 (2009).

[24] Seddon K. R., Stark A. and Torres M. J., Pure Appl. Chem., 72, 2275 (2000).

[25] Slattery J. M., Daguenet C., Dyson P. J., Schubert T. J. S. and Krossing I., Angew. Chem. Int. Ed., 46, 5384 (2007).

[26] Tsukasa T., Tsuda T., Okazaki K. and Kuwabate S., Adv. Mater., 22, 1196 (2010).

[27] Keskin S., Kayrak-Talay D., Akman U. and Hortacsu Ö., J. of Supercritical Fluids, 43, 150 (2007).

[28] Clavel G., Larionaova J., Guari Y. and Guerin Ch., Chem. Eur. J., 12, 3798 (2006).

[29] Guerrero-Sánchez C., Erdmenger T., Sereda P., Wouters D. and Schubert U. S., Chem. Eur. J., 12, 9036 (2006).

[30] Gómez-Ramírez A., López-López M. T., González-Caballero F. and Durán J. D. G., Smart Mater. Struct. 20, 045001 (2011).

[31] Dodbiba G., Park H. S., Okaya K. and Fujita T., J. Magn. Magn. Mater., 320, 1322 (2008).

[32] Guerrero-Sánchez C., Ortiz-Alvarado A. and Schubert U. S., 11th Inter. Conf. on Electrorheological Fluids and Magnetorheological Suspensions, Journal of Physics: Conference Series, 149, 12052 (2009).

[33] Oliveira F. C., Rossi L. M., Jardim R. F. and Rubim J. C., J. Phys. Chem. C, 113, 8566 (2009).

[34] Ueno K., Inaba A., Kondoh M. and Watanabe M., Langmuir, 24, 5253 (2008). 
[35] Khare V., Kraupner A., Mantion A., Jelicic A., Thünemann A. F., Giordano C. and Taubert A., Langmuir, 26, 10600 (2010).

[36] Rodriguez-Arco L., López-López M. T., González-Caballero F. and Durán J. D. G., J. Colloid Inter. Sci. 357, 252-254 (2011).

[37] Barnes H. A., J. Non-Newtonian Fluid Mech., 56, 221 (1995).

[38] Buscall R., J. Rheol., 54, 1177 (2010).

[39] Buscall R., McGowan J. I. and Morton-Jones A. J., J. Rheol., 37, 621 (1993).

[40] Gregory T. and Mayers S., Surface Coating Int. (JOCCA), 76, 82 (1993).

[41] Isa L., Besseling R., and Poon W. C. K., Phys. Rev. Lett., 98, 198305 (2007).

[42] Aral B. K. and Kalyon D. M., J. Rheol., 38, 957 (1994).

[43] Persello J., Magnin A., Chang J., Piau J. M., and Cabane B., J. Rheol., 38, 1845 (1994).

[44] Pignon F., Magnin A. and Piau J. M., J. Rheol., 40, 573 (1996).

[45] Russel W. B. and Grant M. C., Colloids Surf. A, 161, 271 (2000).

[46] Walls H. J., Caines S. B., Sanchez A. M. and Khan S. A., J. Rheol., 47, 847 (2003).

[47] Laun H. M., Gabriel C. and Schmidt G., J. Non-Newtonian Fluids Mech., 148, 47 (2008).

[48] López-López M. T., Kuzhir P., Durán J. D. G. and Bossis G., J. Rheol, 54, 1119 (2010).

[49] Larson, R. G., The Structure and Rheology of Complex Fluids (Oxford University Press, New York, 1999).

[50] Barnes H. A., J Rheol., 33, 329 (1989).

[51] Volkova O., Bossis G., Guyot M., Bashtovoi V. and Reks A., J. Rheol., 44, 91 (2000).

[52] McLeish T. C. B., Jordan T. and Shaw M. T., J. Rheol., 35, 427 (1991).

[53] Klingenberg D. J., J. Rheol., 37, 199 (1993).

[54] Jordan T. C., Shaw M. T. and McLeish T. C. B., J. Rheol., 36, 441 (1992).

[55] Parthasarathy M., Ahn K. H., Belongia B. M. and Klingenberg D. J, Int. J. Mod. Phys. B, 8, 2789 (1994).

[56] Parthasarathy M. and Klingenberg D. J., Mater. Sci. Eng., 17, 57 (1996). 